\title{
Field theory of statics and dynamics of glasses: rare events and barrier distributions
}

\author{
Leon Balents ${ }^{1}$ and Pierre Le Doussal ${ }^{2}$ \\ ${ }^{1}$ Department of Physics, University of California, Santa Barbara, CA 93106-4030 \\ ${ }^{2}$ CNRS-Laboratoire de Physique Théorique de l'Ecole Normale Supérieure, 24 Rue Lhomond 75231 Paris, France
}

(Dated: November 21, 2018)

\begin{abstract}
We study thermally activated dynamics using functional renormalization within the field theory of randomly pinned elastic systems, a prototype for glasses. It appears through an essentially nonperturbative boundary layer in the running effective action, for which we find a consistent scaling ansatz to all orders. We find that in the statics the boundary layer describes the physics of rare low energy metastable states as suggested by a phenomenological droplet picture. The extension to equilibrium dynamics mandates a non-trivial broad distribution of relaxation times for the large scale modes, hitherto neglected in analytic calculations.
\end{abstract}

Extremely slow dynamics and dominance of rare fluctuations are ubiquitous properties of complex and disordered materials. Such behavior occurs in a panoply of systems involving many interacting degrees of freedom, from spin glasses to supercooled liquids to the pinned elastic medium. We study the latter here as a prototype due to its relative simplicity. It describes numerous experimental systems such as interfaces in magnets [1, 2, charge density waves and Wigner crystals [3], or vortex lattices in superconductors 肺. The elastic medium is described by a displacement field $\Phi(\mathbf{r})$, with Hamiltonian

$$
H[u]=\int d^{d} \mathbf{r}\left[\frac{1}{2}|\nabla \Phi|^{2}+V(\Phi(\mathbf{r}), \mathbf{r})\right],
$$

where $V(\Phi, r)$ is a zero-mean Gaussian random potential with variance $\overline{V(\Phi, r) V\left(\Phi^{\prime}, r^{\prime}\right)}=R\left(\Phi-\Phi^{\prime}\right) \delta^{(d)}\left(r-r^{\prime}\right)$ (an overline indicates disorder averaging). Different physical cases are described by varying longitudinal dimensionality $(d)$, transverse dimensionality $(N=$ the number of vector components of $\Phi$ - we take $N=1$ for simplicity here), and $R(\Phi)$. The Langevin equation of motion is

$$
\eta \partial_{t} \Phi_{r t}=\nabla_{r}^{2} \Phi_{r t}+f\left(\Phi_{r t}, r\right)+\zeta(r, t),
$$

with friction $\eta$, thermal noise $\zeta(r, t)$ and random force $f(\Phi, r)=-\partial V(\Phi, r) / \partial \Phi$ (denoting $\left.\Phi(r, t) \equiv \Phi_{r t}\right)$.

A minimal physical description of disordered glasses is provided by the droplet scenario $(5$. In its simplest form, it supposes the existence, at each length scale $L$, of a small number of excitations of size $\delta \Phi \sim L^{\zeta}$ above a ground state, drawn from an energy distribution of width $\delta E \sim L^{\theta}$ with constant weight near $\delta E=0$. Although the applicability of the droplet picture to more complex systems such as spin glasses is controversial, it appears to describe simpler models such as Eq. (1) relatively well, at least in low dimensions, [6] and we summarize its main conclusions. Static thermal fluctuations at a given scale are dominated by rare samples/regions with two nearly degenerate minima. In particular, the $(2 n)^{\text {th }}$ moment of $\Phi$ fluctuations is expected to behave as

$$
\overline{\left(\left\langle\Phi^{2}\right\rangle-\langle\Phi\rangle^{2}\right)^{n}} \sim c_{n}\left(T / L^{\theta}\right) L^{2 n \zeta} .
$$

The droplet picture supposes the long-time equilibrium dynamics is dominated by thermal activation between these quasi-degenerate minima controlled by barriers of typical scale $U_{L} \sim L^{\psi}$. Little is known about the distribution of these barriers, but there is some evidence [7] that $\psi \approx \theta$. Even a modest distribution of barriers, however, due to the Arrhenius law $\tau_{L} \sim e^{U_{L} / T}$, yields relaxation time scales with an extremely broad distribution as $T \rightarrow 0$. An important unanswered question is the behavior of relaxation time moments, defined e.g. by

$$
\overline{\tau_{L}^{n}}=\overline{\frac{\int_{0}^{\infty} d t t^{n} R_{q, t}}{\int_{0}^{\infty} d t R_{q, t}}} \sim \int d U_{L} p\left(U_{L}\right) e^{n U_{L} / T},
$$

with $q=L^{-1}$ and the response function $R_{q, t-t^{\prime}}=$ $\partial \Phi_{q}(t) / \partial \zeta_{q}\left(t^{\prime}\right)$. The moment $\overline{\tau_{L}^{n}}$ may behave as $\overline{\tau_{L}^{n}} \sim$ $e^{\alpha(n) U_{0} L^{\theta} / T}$ with $\alpha(n) \geq n$, or grow even faster with $\ln \overline{\tau^{n}} \gg L^{\theta}$. A theory of these timescales is crucial to understanding both equilibrium response and correlations and to near-equilibrium phenomena such as creep [2, 4].

Despite the wide applicability and extensive theoretical studies of the elastic model, Eqs. (112), analytic treatment for $d \geq 1$ of the non-zero temperature glassy behavior has been limited to mean-field $(N \rightarrow \infty)$ 8, 9] and infinite dimension $(d \rightarrow \infty)$ limits 10 in which thermal activation over divergent barriers $\left(U_{L} \sim L^{\theta}\right)$ is not included. The existence of these growing barriers appears to be captured by the field-theoretic Functional Renormalization Group (FRG) method 11, 12, 13. (for $\epsilon=4-d \ll 1$ ), extended to non zero temperature 14, 15, but until now neither the rare events nor fluctuating barriers have been obtained in this approach.

In this paper, we investigate how properties reminiscent of droplets can emerge naturally from the FRG. Although temperature is formally irrelevant (in the RG sense), thermal effects give rise to an intricate non-perturbative boundary layer structure. Nevertheless, a consistent scaling of this boundary layer can be understood, providing a field-theoretic framework for thermally-activated glassy dynamics. This structure appears to support solutions of the droplet type, with in particular rare thermal fluctuations obeying Eq. (3) and 
a distribution of timescales $\overline{\tau^{n}}$ described by a non-trivial function $\alpha(n)$. Details can be found in $[16]$.

We begin by considering the statics. The theory is formulated in terms of the disorder-averaged partition function for $n$ replicas $\vec{\Phi}=\left(\Phi_{1}, \cdots, \Phi_{n}\right)$,

$$
Z_{s}=\int D \vec{\Phi} \exp \left\{-\int_{r}\left[\sum_{a} \frac{1}{2 T}\left|\nabla \Phi_{a}\right|^{2}-U[\vec{\Phi}(r)]\right]\right\} .
$$

A more general distribution for $V(\Phi, r)$ (still independently distributed at each $r$ ) is expressed through the characteristic function $U[\vec{\Phi}]=\sum_{k=2}^{\infty} \frac{1}{T^{k} k !} \sum_{a_{1} \cdots a_{k}} S^{(k)}\left[\Phi_{a_{1}}, \cdots, \Phi_{a_{k}}\right]$. Here $S^{(k)}\left(\Phi_{1}, \cdots, \Phi_{k}\right)$ is the $k^{\text {th }}$ connected cumulant of $V(\Phi, r)$ at fixed $r$ (note $S^{(2)}\left(\Phi, \Phi^{\prime}\right)=R\left(\Phi-\Phi^{\prime}\right)$ ).
By translational invariance, these cumulants satisfy $S^{(k)}\left(\Phi_{1}+\lambda, \cdots, \Phi_{k}+\lambda\right)=S^{(k)}\left(\Phi_{1}, \cdots, \Phi_{k}\right)$, so $U[\vec{\Phi}]$ is a fully symmetric, translationally-invariant function of $\vec{\Phi}$. As is well-known [11], zero-temperature power-counting shows that all $S^{(k)}$ for $k>2$ are irrelevant, and the second cumulant $R\left(\Phi-\Phi^{\prime}\right)$ is marginally relevant just below $d=4$. One therefore finds that for $\epsilon=4-d \ll 1$, the fixed point function $R^{*}(\Phi) \sim \epsilon \ll 1$ and $\zeta$ also of $O(\epsilon)$. This smallness justifies the truncation $S^{(k)} \approx 0$ for $k>2$ at one-loop, to leading order in $\epsilon$ at $T=0$.

Prior investigations of thermal effects have presumed this truncation remains valid at $T>0$ 14, 15. We check this assumption by considering the corrections due to $S^{(3)} \neq 0$. A straightforward Wilson scheme calculation keeping only $k=2,3$ terms to one loop gives

$$
\begin{aligned}
& \partial_{l} \tilde{R}(\Phi)=\left(\epsilon-4 \zeta+\zeta \Phi \partial_{\Phi}\right) \tilde{R}(\Phi)+\bar{T}_{l} \tilde{R}^{\prime \prime}(\Phi)+\tilde{S}_{110}^{(3)}(0,0, \Phi)+\frac{1}{2} \tilde{R}^{\prime \prime}(\Phi)^{2}-\tilde{R}^{\prime \prime}(0) \tilde{R}^{\prime \prime}(\Phi) \\
& \partial_{l} \tilde{S}^{(3)}\left(\Phi_{123}\right)=\left(2 \epsilon-2-6 \zeta+\zeta \Phi_{i} \partial_{\Phi_{i}}\right) \tilde{S}^{(3)}\left(\Phi_{123}\right)+\operatorname{sym}\left(\frac{3 \bar{T}_{l}}{2} \tilde{S}_{200}^{(3)}\left(\Phi_{123}\right)+\frac{3 \bar{T}_{l}}{2} \tilde{R}^{\prime \prime}\left(\Phi_{13}\right) \tilde{R}^{\prime \prime}\left(\Phi_{23}\right)\right) \\
& +3 \operatorname{sym}\left(\tilde{R}^{\prime \prime}\left(\Phi_{12}\right)\left(\tilde{S}_{110}^{(3)}\left(\Phi_{113}\right)-\tilde{S}_{110}^{(3)}\left(\Phi_{123}\right)\right)+\tilde{R}^{\prime \prime}\left(\Phi_{12}\right) \tilde{\mathrm{R}}^{\prime \prime}\left(\Phi_{13}\right)^{2}-\frac{1}{3} \tilde{\mathrm{R}}^{\prime \prime}\left(\Phi_{12}\right) \tilde{\mathrm{R}}^{\prime \prime}\left(\Phi_{23}\right) \tilde{\mathrm{R}}^{\prime \prime}\left(\Phi_{31}\right)\right)
\end{aligned}
$$

where $\Lambda_{l}=\Lambda e^{-l}$ is the running cutoff, $\Phi_{i j}=\Phi_{i}-\Phi_{j}$, $\Phi_{i j k}=\Phi_{i}, \Phi_{j}, \Phi_{k}$. With $A_{d}=1 /\left(2^{d-1} \pi^{d / 2} \Gamma(d / 2)\right)$, the rescaled functions $\tilde{R}(\Phi)=A_{d} \Lambda_{l}^{d-4+4 \zeta} R\left(\Phi \Lambda_{l}^{-\zeta}\right)$ and $\tilde{S}^{(3)}\left(\Phi_{123}\right)=A_{d}^{2} \Lambda_{l}^{2 d-6+6 \zeta} S^{(3)}\left(\Phi_{123} \Lambda_{l}^{-\zeta}\right)$. The ratio of thermal energy $T$ to pinning energy $L^{\theta}$ defines $\bar{T}_{l}=$ $A_{d} T \Lambda_{l}^{\theta}$, where $\theta=d-2+2 \zeta>0$ indicates the dominance of energy over entropy as appropriate for a glass. It was convenient to define $\tilde{R}(\Phi)=\tilde{R}(\Phi)-\tilde{R}^{\prime \prime}(0) \Phi^{2} / 2$, since $\tilde{R}^{\prime \prime}(0)$ does not feed into higher cumulants.

The naive $T>0$ analysis assumes $\tilde{S}^{(3)} \ll \epsilon \tilde{R}$ to convert Eq. (6) into a decoupled equation for $\tilde{R}(\Phi)$. Its solution yields two regimes. For $\Phi \sim O(1) \gg \bar{T}_{l}$, the solution $\tilde{R}_{l}(\Phi)$ converges to a zero temperature fixed point function [11], with a small set of universality classes. [4, 14] For, e.g. $V(\Phi, x)$ periodic on the interval $0<\Phi<1$, the force-force correlator $\tilde{\Delta}(\Phi)=-\tilde{R}^{\prime \prime}(\Phi)=\operatorname{Min}_{n \in \mathcal{Z}} \frac{\epsilon}{6}[(\Phi-$ $\left.n-1 / 2)^{2}-1 / 12\right]$. Non-analytic behavior at small $\Phi$, $\tilde{\Delta}_{T=0}(\Phi)-\tilde{\Delta}(0) \sim-\chi|\Phi|\left(\chi \equiv\left|\tilde{\Delta}^{\prime}\left(0^{+}\right)\right|\right)$is, however, super-universal, i.e. the same for all disordered elastic models. The significance of this cusp has been discussed by several authors. 9, 11, 17] Notably, the fluctuations of the mean curvature of the pinning potential, $-\tilde{\Delta}_{T=0}^{\prime \prime}(0)=\overline{\left(\partial_{\Phi}^{2} V(\Phi)\right)^{2}}=+\infty$, which implies the existence of multiple metastable minima in the effective potential. We will see further consequences below. In this $T=0$ regime, the neglect of $S^{(3)}$ appears consistent: solution of Eq. (7) gives $\tilde{S}_{T=0}^{(3)} \sim O\left(\epsilon^{3}\right) \ll \tilde{R}_{T=0}$, yielding only higher-order corrections.

The convergence to this solution is non-uniform, how- ever, and for $\Phi \lesssim \epsilon \bar{T}_{l}$, the cusp singularity is rounded in a thermal boundary-layer. The boundary layer form follows from Eq. (6) for $\hat{S}^{(3)}=0$ through the ansatz $\tilde{\mathrm{R}}(\Phi)=-\bar{T}_{l}^{3}(\epsilon \tilde{\chi})^{-2} r(\phi)$, with $\tilde{R}^{\prime \prime}(0)=\tilde{\chi}^{2} \epsilon^{2} /(2 \zeta-\epsilon)$, keeping $\phi=\epsilon \tilde{\chi} \Phi / \bar{T}_{l}$ fixed of $O(1), \tilde{\chi}=\chi / \epsilon$ and $\bar{T}_{l} \ll \epsilon^{2}$. This gives $r^{\prime \prime}(\phi)=1-\sqrt{1+\phi^{2}}$, 15 matching the $|\phi|$ cusp as $\phi \rightarrow \infty$, but becoming smooth for $\phi$ of $O(1)$.

Even within the one loop Wilson RG, however, the neglect of the third cumulant is unjustified in the boundary layer. This follows since the $O\left[\left(\mathrm{R}^{\prime \prime}\right)^{3}\right]$ terms in Eq. (7) feed their boundary-layer scaling forms into $S^{(3)}$. Naively balancing these terms with the rescaling part $\left(\approx-2 \tilde{S}^{(3)}\right)$ suggests $\tilde{S}^{(3)} \sim \bar{T}_{l}^{3}$. This is, however, inconsistent, since the remaining terms linear in $\tilde{S}^{(3)}$ would then be $O\left(\bar{T}_{l}^{2}\right)$. Instead, the only consistent ansatz is $\tilde{S}^{(3)}\left(\Phi_{1}, \Phi_{2}, \Phi_{3}\right)=(\epsilon \tilde{\chi})^{-2} \bar{T}_{l}^{4} s^{(3)}\left(\phi_{1}, \phi_{2}, \phi_{3}\right)$. This in turn generates a feedback in Eq. (6) that is of the same order as the other surviving terms in the boundary layer.

In fact, the scaling forms in Eqs. (6-7) must be extended to all cumulants. Defining $U(\vec{\Phi})=A_{d} \Lambda_{l}^{d} \tilde{U}\left(\vec{\Phi} \Lambda_{l}^{\zeta}\right)$, then $\tilde{U}(\vec{\Phi})=\sum_{p,\left\{a_{i}\right\}} f_{2 p} \phi_{a_{1}} \cdots \phi_{a_{2 p}}+\frac{\bar{T}_{l}}{(\epsilon \tilde{\chi})^{2}} u(\vec{\phi})$, with $f_{2 p}=\tilde{\Delta}^{(2 p)}(0) /\left[(2 p) !(\tilde{\chi} \epsilon)^{2 p}\right]$, where $\tilde{\Delta}^{(2 p)}(0)$ is the $(2 p)^{\text {th }}$ cumulant of the random force $\left(\tilde{\Delta}^{(2)}(0)=\Delta(0)\right)$, and the boundary-layer replica vector $\vec{\phi}_{l}=\epsilon \tilde{\chi} \vec{\Phi} / \bar{T}_{l}$. This gives the non-trivial one-loop Wilson fixed point equation,

$$
\sum_{p,\left\{a_{i}\right\}} x_{2 p} f_{2 p} \phi_{a_{1}} \cdots \phi_{a_{2 p}}+\operatorname{Tr} \ln \left[\delta^{a b}-\partial_{a} \partial_{b} u(\vec{\phi})\right]=0,
$$


with scaling eigenvalues $x_{2}=\epsilon-2 \zeta$ of $O(\epsilon)$ and $x_{2 p}=$ $d-2 p(\theta-\zeta)$ of $O(1)$ for $p>1$. Expansion of Eq. (8) in number of replica sums gives coupled boundary layer equations for $r$ and $s^{(3)}$, and all higher cumulants.

Unfortunately, the disappearance of $\epsilon$ from Eq. (8) (since $x_{2 p} f_{2 p} \sim O(1)$ ), indicates that a one-loop analysis may be insufficient. Worse, higher loop corrections to $U$ appear naively extremely singular in $1 / T$ : each additional contraction carries with it two $\partial_{\Phi}$ derivatives, and hence two factors of $1 / T$, but only a single propagator proportional to $T$, so increasing powers of $1 / T$ appear in higher loop diagrams. This makes the non-perturbative status of the boundary layer extremely nontrivial. To verify its existence, we therefore employ the Exact RG (ERG) method [18], taking from now on $\zeta=0$ for simplicity. The ERG extends the characteristic function $U(\vec{\Phi})$ to a non-local functional, $\mathcal{U}[\vec{\Phi}(r)]$, defined by a running effective action $\Gamma(\vec{\Phi})=(1 / 2) \Phi: G_{l}^{-1}: \Phi+\mathcal{U}_{l}[\vec{\Phi}]$, with a cutoff Green's function $\left(G_{l}\right)_{a b}(q)=\delta_{a b}\left(T / q^{2}\right)\left(c\left(\frac{q^{2}}{\Lambda^{2}}\right)-c\left(\frac{q^{2}}{\Lambda_{l}^{2}}\right)\right)$. Then

$$
\partial_{l} \mathcal{U}_{l}(\vec{\Phi})=\frac{1}{2} \operatorname{Tr} \partial_{l} G_{l}:\left(G_{l}^{-1}-G_{l}^{-1}\left(1+G_{l}: \frac{\delta^{2} \mathcal{U}_{l}}{\delta \Phi \delta \Phi}\right)^{-1}\right),
$$

where $\delta^{\prime} s$ indicate functional differentiation. This equation can be expanded order by order in $\mathcal{U}_{l}$ to generate a series expansion. The functional nature of $\mathcal{U}[\vec{\Phi}(r)]$ necessitates a further multilocal expansion $\mathcal{U}_{l}[\vec{\Phi}]=\sum_{p=1}^{\infty} \int_{x_{1} \cdots x_{p}} U_{l}^{(p)}\left(\vec{\Phi}_{x_{1}} \cdots \vec{\Phi}_{x_{p}} ; x_{1} \cdots x_{p}\right)$, in terms of translationally-invariant functions ("p-local terms") of $p \Phi_{i}$ and $x_{i}$ variables. Remarkably, exact integro-differential flow equations for the $U_{l}^{(p)}$ can be obtained. The boundary layer ansatz, applied to each p-local term generalizes to $U_{l}^{(p)}\left(\left\{\vec{\Phi}_{i}\right\},\left\{x_{i}\right\}\right)=$ $\epsilon^{-2} \Lambda_{l}^{p d} \bar{T}_{l} u_{l}^{(p)}\left(\left\{\vec{\phi}_{i l}\right\},\left\{\Lambda_{l} x_{i}\right\}\right)$, plus a nonlocal extension of the random-force terms $\left(f_{2 p}\right)$. Inserting this into Eq. (9), one finds that apart from the random-force terms, the left-hand-side is negligible for $\bar{T}_{l} \ll 1$ and $\vec{\phi} \sim O(1)$. This gives a non-trivial hierarchy of integro-differential equations (too complicated to present here) for the scaling functions $u_{l}^{(p)}$ with a well-defined limit as $l \rightarrow \infty$. We have checked that the truncation to $p \leq 2$ is non-singular [16], i.e. the $p=2$ term can be eliminated to obtain a single equation for the local function $u_{l}^{(1)}$ involving no divergent integrals. We believe this procedure, which effectively resums the $1 / T$ divergences into a non-singular result, can be carried out systematically to all orders.

This indicates the non-perturbative consistency of the boundary layer ansatz in the ERG. We now turn to some physical implications. A tree level calculation gives

$$
\overline{\left(\left\langle\Phi^{2}\right\rangle-\langle\Phi\rangle^{2}\right)^{n}} \sim T^{2 n} \partial_{\left\{\Phi_{a}\right\}}^{2 n} U(0) \int d^{d} \mathbf{r}^{\prime} G^{2 n}\left(\mathbf{r}^{\prime}\right),
$$

where $\partial_{\left\{\Phi_{a}\right\}}^{2 n} U$ is a combination of $2 n^{\text {th }}$ order derivatives of $U(\vec{\Phi})$, and $G\left(\mathbf{r}^{\prime}\right)=\int d^{d} \mathbf{q} / q^{2} \sim\left|r^{\prime}\right|^{2-d}$. Remarkably, inserting the boundary layer scaling form precisely reproduces the droplet behavior of thermal fluctuations, Eq. (3), with $c_{n} \sim \partial_{\left\{\phi_{a}\right\}}^{2 n} u(0)$ ! A further test of droplet predictions is that the simplest three-well quantity $C_{3}=$ $\left\langle\left(\Phi_{a}-\Phi_{b}\right)^{2}\left(\Phi_{b}-\Phi_{c}\right)^{2}\left(\Phi_{a}-\Phi_{c}\right)^{2}\right\rangle$ vanishes to $O(T)$. We have verified $C_{3} \sim O\left(T^{2}\right)$ for $d=0, N=1, \theta>0$. Some further results 16 agree with recent calculations in this case where the simplest droplet picture is exact [19].

We now turn to the dynamics. Prior investigations 15] have calculated the renormalization of the mean relaxation time at $O(\tilde{\Delta}), \partial_{l} \bar{\eta}_{l}=-\tilde{\Delta}^{\prime \prime}(0) \bar{\eta}_{l}$, leading through the boundary layer scaling $\tilde{\Delta}^{\prime \prime}(0) \sim 1 / \bar{T}_{l}$ to $\bar{\eta}_{l}=\eta_{0} \exp \left[\frac{\chi^{2}}{\theta}\left(1 / \bar{T}_{l}-1 / \bar{T}_{0}\right)\right]$ in Eq. (2). This is consistent with an energy barrier $U_{L}=U_{0} L^{\theta}$ (with $L=\Lambda_{l}^{-1}$ ).

We begin our consideration of the distribution of relaxation times in a simplified calculation generalizing Eq. (2) to a random friction coefficient $\eta \rightarrow$ $\eta(r)$, where the fluctuation-dissipation theorem requires $\left\langle\zeta(r, t) \zeta\left(r^{\prime}, t^{\prime}\right)\right\rangle=2 \eta(r) T \delta^{d}\left(r-r^{\prime}\right) \delta\left(t-t^{\prime}\right)$. We take $\eta(r)$ with an independent and identical distribution $P(\eta)$ at each $r$, with the characteristic function $F(z)=$ $-\ln \int_{0}^{+\infty} d \eta P(\eta) e^{-z \eta}$. In the standard Martin-SiggiaRose (MSR) formalism, thermal and disorder averages over the equation of motion are calculated from a functional integral over $\Phi_{r t}, \hat{\Phi}_{r t}$ with the measure $\exp \left(-S_{0}-\right.$ $\left.S_{\mathrm{st}}-S_{\mathrm{dy}}\right)$, where $S_{0}=\int_{r t}-i \hat{\Phi}_{r t} \nabla_{r}^{2} \Phi_{r t}$,

$$
\begin{aligned}
S_{\mathrm{st}} & =\sum_{n} \frac{-1}{n !} \int_{r t_{1} \cdots t_{n}}\left(i \hat{\Phi}_{r t_{n}} \cdots i \hat{\Phi}_{r t_{n}}\right) \Delta^{(n)}\left(\Phi_{r t_{1}}, \cdots, \Phi_{r t_{n}}\right)(11) \\
S_{\mathrm{dy}} & =\int_{r} F\left[\int_{t}\left(i \hat{\Phi}_{r t} \partial_{t} \Phi_{r t}-T i \hat{\Phi}_{r t} i \hat{\Phi}_{r t}\right)\right]
\end{aligned}
$$

and $\Delta^{(n)}(\cdot)=(-1)^{n} \partial_{\Phi_{1}} \cdots \partial_{\Phi_{n}} U^{(n)}(\cdot)$. Setting $F(z)=$ $\bar{\eta} z$ recovers the previous approach, but when $F(z)$ is nontrivial Eqs. (11.12) comprise an unusual dynamical field theory. First consider the random friction model with $\Delta^{(n)}=U^{(n)}=0$. Despite its highly non-quadratic action, this represents an FRG fixed manifold, with nontrivial response function moments, indexed by the "Fterm" $F(z)$, which remains un-renormalized. This follows since Eq. (2) becomes linear for zero pinning force $f=0$, hence the distribution of $\eta(r)$ is scale-independent.

This changes drastically in the presence of pinning disorder $\Delta^{(n)} \neq 0$. Considering for the moment only the second cumulant $n=2$, to $O(\Delta) F(z)$ is no longer scaleindependent: $\partial_{l} F_{l}[z]=\left(\partial_{l} \ln \bar{\eta}_{l}\right)\left(z F_{l}^{\prime}[z]+2 z^{2} F_{l}^{\prime \prime}[z]\right)$. This implies the rapid growth of higher connected cumulants of $\eta$, to wit $F(z)=-\sum_{q} \frac{(-1)^{q}}{q !} \eta^{(q)} z^{q}$, with

$$
\eta_{l}^{(q)} \sim \eta_{0}^{(q)} e^{\chi^{2} \alpha(q) / \bar{T}_{l} \theta}=\eta_{0}^{(q)}\left(\bar{\eta}_{l} / \eta_{0}\right)^{\alpha(q)} \gg\left(\bar{\eta}_{l}\right)^{q},
$$

where $\alpha(q)=2 q^{2}-q \cdot[16,20$ Thus all the renormalized moments of the friction have the Arrhenius form, but since $\alpha(q)>q$, they cannot be characterized by a single relaxation time $\bar{\eta}_{l}$. The quadratic form of $\alpha(q)$ obtained 
in this approximation is consistent with a very broad, lognormal distribution of relaxation times $\eta$. The more complete analysis below provides a consistent ansatz which extends this scaling to the full effective action, with, however, non-trivial exponents $\alpha(q)$. 21

To go beyond this simplified picture, we must consider all interaction vertices at a given "level" $q$ involving a fixed number $q$ of total time derivatives (power of frequency $\omega^{I}$ ), which includes e.g. $F^{(q)}[0]$ but also an infinite hierarchy of higher cumulant functions, as in the static case $q=0$. An examination of the oneloop low-temperature FRG shows that for each value of $q$ one obtains a distinct hierarchy such that all terms grow proportionately to $\eta_{l}^{(q)}$ with a non-trivial exponent $\alpha(q)$. The hierarchies at different $q$ are coupled in a special manner such that only levels $q^{\prime}<q$ feed into level $q$. This structure allows for independent exponents $\alpha(q)$ provided they satisfy the natural convexity constraint $\alpha(q)>\alpha\left(q^{\prime}\right)+\alpha\left(q-q^{\prime}\right)$ for $1 \leq q^{\prime} \leq q-1$. Each member of the hierarchy scales as $\eta_{l}^{(q)}$ times a distinct scaling function in the boundary layer regime $\phi \sim O(1)$. Coupled differential eigenvalue equations involving these functions determine the exponents $\alpha(q)$ by matching.

We now illustrate this structure for $q=1$, calculating the FRG for terms up to second cumulant order:

$$
\begin{aligned}
S_{\mathrm{dy}} & =\int_{r t} \bar{\eta} i \hat{\Phi}_{r t} \dot{\Phi}_{r t}-\int_{r t_{1} t_{2}} i \hat{\Phi}_{t_{1}} i \hat{\Phi}_{r t_{2}} \dot{\Phi}_{r t_{1}} G\left(\Phi_{r t_{1}}-\Phi_{r t_{2}}\right) \\
& -\frac{1}{6} \int_{r t_{1} t_{2} t_{3}} i \hat{\Phi}_{r t_{1}} i \hat{\Phi}_{r t_{2}} i \hat{\Phi}_{r t_{3}} \dot{\Phi}_{r t_{1}} H\left(\Phi_{r t_{1}}, \Phi_{r t_{2}}, \Phi_{r t_{3}}\right)(14
\end{aligned}
$$

where $G(-\Phi)=-G(\Phi)$. One finds that $G$ contributes to the renormalization of $\bar{\eta}$ :

$$
\partial_{l} \bar{\eta}=\left(\bar{G}^{\prime}(0)-\tilde{\Delta}^{\prime \prime}(0)\right) \bar{\eta}
$$

where $G(\Phi)=\bar{\eta}_{l} \Lambda_{l}^{2-d} A_{d}^{-1} \bar{G}(\Phi)$, correcting the naive $\tilde{\Delta}^{\prime \prime}(0)$ contribution to $\bar{\eta}$. Although $\bar{G}$ is nominally irrelevant, $\partial_{l} \bar{G}=(-2+\epsilon) \bar{G}+\cdots$, it supports a non-trivial boundary layer, in which $\bar{G}(\Phi)=\epsilon \tilde{\chi} g(\phi)$, satisfying

$$
\begin{gathered}
0=2 f^{\prime \prime} g+(1+f) g^{\prime \prime}-g^{\prime}(0)\left(g-f^{\prime}\right)+2 f^{\prime}\left(f^{\prime \prime}(0)+f^{\prime \prime}\right) \\
+3 g^{\prime} f^{\prime}+s_{221}^{(3)}(\phi, \phi, 0)+2 h_{100}(0, \phi, 0)+\frac{2}{3} h_{010}(0, \phi, 0),
\end{gathered}
$$

where $f(\phi)=-r^{\prime \prime}(\phi), H\left(\Phi_{123}\right)=\bar{\eta}_{l} \Lambda_{l}^{4-2 d} A_{d}^{-1} \epsilon^{2} h\left(\phi_{123}\right)$. Matching to the well-behaved "outer" solution requires $g(\phi) \rightarrow_{\phi \rightarrow \infty} g_{+\infty}$, a constant, while for $\phi \ll 1, g(\phi) \sim$ $g^{\prime}(0) \phi$. For given functions $f, s^{(3)}, h$, this equation is an eigenvalue problem for $g^{\prime}(0)$ (indeed, analysis shows that $g^{\prime}(0)$ must be tuned to match the outer solution). From Eq. (15), the exponent $\alpha(1)=g^{\prime}(0)+f^{\prime \prime}(0)$ is thereby non-trivial as claimed. Moreover, $\alpha(1)$ is determined through the above equation in a manner depending upon the full boundary layer functions $r, s, h$. Similar considerations apply to $q=2$ (details suppressed to save space), for which the one-time term is $D \int_{r t} \hat{u} \ddot{u}$, and there are 3 independent functions at the second cumulant level. The $q=1$ functions feed into the $q=2$ hierarchy in such a way that the $\alpha(2)$ exponent governing the growth of $D$ is independent provided $\alpha(2)>2 \alpha(1)$ self-consistently.

In summary, we have described a consistent nonperturbative boundary layer structure in a static field theory for pinned elastic systems, successfully describing rare events dominating low-temperature thermal fluctuations. An extension to the dynamics leads to a broad distribution of relaxation times, for which the moments $\overline{\tau^{n}}$ retain a (non-trivial) Arrhenius form. This condition would appear required for simple scaling arguments for creep to be even qualitatively correct, and is experimentally testable. Our investigations uncover an intimate connection between the highly non-perturbative boundary layer and droplet considerations, suggesting that an analytic approach encapsulating this physics might be successful in the near future. The more general applicability of these ideas to more complex glassy systems such as spin and structural glasses, as well as to the quantum regime, appears as a distinct possibility.

L.B. thanks L. Radzihovsky and D. S. Fisher for inspiration. L.B. was supported by NSF grants DMR9985255,INT-0089835, and the Sloan and Packard foundations.

[1] J.P.Bouchaud et al. and T. Nattermann Spin glasses and random fields Ed. A. P. Young, World Scientific, Singapore, 1998.

[2] S. Lemerle et al. Phys. Rev. Lett. 80 (1998) 849.

[3] G. Grüner, Rev. Mod. Phys. 60, 1129 (1988). E. Y. Andrei and al., Phys. Rev. Lett. 60, 2765 (1988).

[4] G. Blatter et al. Rev. Mod. Phys. 661125 (1994); T. Giamarchi and P. Le Doussal, Phys. Rev. B52 1242 (1995).

[5] D.S. Fisher, D.A. Huse Phys. Rev. B 38373 (1988).

[6] See, e.g. A. A. Middleton, Phys. Rev. B63 60202 (2001); T. Hwa and D. S. Fisher, Phys. Rev. B49, 3136 (1994).

[7] B. Drossel and M. Kardar, Phys. Rev. E 52, 4841 (1995). L. V. Mikheev, B. Drossel, and M. Kardar, Phys. Rev. Lett. 75, 1170 (1995).

[8] M. Mézard, G. Parisi J. Phys. I (France) 1809 (1991). L. Cugliandolo et al. Phys. Rev. Lett. 762390 (1996).

[9] P. Le Doussal, K.J. Wiese, cond-mat/0109204.

[10] D.S. Fisher Phys. Rev. B 311396 (1985); J. Vannimenus, B. Derrida J. Stat. Phys. 1051 (2001).

[11] D. S. Fisher, Phys. Rev. Lett. 56, 1964 (1986), L. Balents, D. S. Fisher, Phys. Rev. B 48, 5959 (1993).

[12] T. Nattermann et al. J. Phys. (Paris) 2, 1483 (1992). O. Narayan, D. S. Fisher, Phys. Rev. B 46, 11520 (1992).

[13] P. Chauve, P. Le Doussal and K.J. Wiese, Phys. Rev. Lett. 86 (2001) 1785.

[14] L. Balents, Europhys. Lett. 24, 489 (1993).

[15] P. Chauve et al. Phys. Rev. B 626241 (2000).

[16] L. Balents, P. Le Doussal in preparation

[17] L. Balents, J. P. Bouchaud, and M. Mézard, J. de Phys. I 6, 1007 (1996).

[18] G. Schehr, P. Le Doussal in preparation, P. Chauve et al. 
Phys. Rev. E 64, 051102 (2001)

[19] P. Le Doussal and C. Monthus, cond-mat/0204168.

[20] D. S. Fisher, L. Radzihovsky, private comm.
[21] In $d=0$ for $N=1$ scaling of $\overline{\tau_{L}^{n}}$ is non-Arrhenius 19, indicating a different scenario there. 